\title{
Diagnosis and education for a better future in Uganda
}

Natasha Kopitsis

Since 2005, the Novo Nordisk Haemophilia Foundation (NNHF; www.nnhf.org) has worked in collaboration with local partners and internationally renowned experts to improve access to haemophilia care. The NNHF has identified three areas on which to focus its activities in order to create impact where it is most needed: capacity building, diagnosis and registry, and education and empowerment. Underpinning these focus areas are targeted awareness creation activities and the development of strategic partnerships, which enable and facilitate local impact creation through empowered community advocates and authority engagement. NNHF supports fellowships, local development projects, and recognises extraordinary achievements via the annual 'NNHF Project of the Year Award' and the 'NNHF Community Award'. Raising awareness of haemophilia in Uganda was the NNHF global fundraiser in 2015. This article summarises how the donations raised were used to establish diagnosis and education for a better future for Uganda's haemophilia patients.

\section{Keywords: Uganda, haemophilia, diagnosis}

A lack of haemophilia awareness in Uganda means many people are not aware they have the condition. Alongside this, until in early 2015, there were no diagnosis facilities in public hospitals, and haemophilia was not on the government's healthcare agenda. Together, these issues meant less than $2 \%$ of people with haemophilia were diagnosed. The Novo Nordisk Haemophilia Foundation (NNHF) initiated a fundraiser to support a project that would address these issues, which began in 2015 under the leadership of Kisakye Agnes executive secretary of the Haemophilia Foundation of Uganda (HFU).

\section{Establishing diagnosis}

To establish diagnosis, the laboratory at Mulago hospital in Kampala was provided with reagents, and other equipment including a coagulometer. In December 2015, Dr Angus McCraw from the United Kingdom delivered a two-day training course to 10 lab technicians, on how to conduct coagulation tests using the reagents and new equipment (Figure 1). Building these new diagnosis facilities and skills means haemophilia testing can now take place at a government hospital, free of charge, for the first time,

Natasha Kopitsis, Communications Manager, Novo Nordisk Haemophilia Foundation. Email: nhhnanovonordisk.com

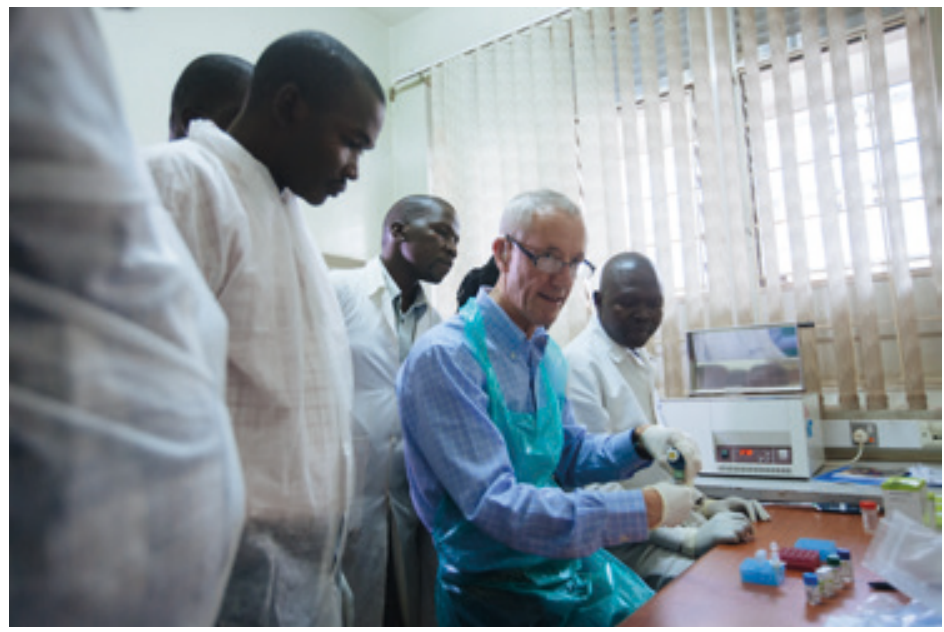

Figure 1: Dr Angus McCraw delivered a two-day practical training course to Mulago's lab technicians

making accurate diagnosis accessible to many Ugandans.

The newly trained laboratory technicians were able to put their learning into practice straightaway, at a screening day taking place at the hospital the following day. During this screening day, potential patients and family members were seen by Dr Kate Khair and members of the haemophilia team from Great Ormond Street Hospital for Children in London. The team participated as part of their World Federation of Haemophilia twinning programme, the activities and goals of which the NNHF aligned with for this project. Those patients describing haemophilia symptoms were screened, leading to 49 new patients being diagnosed (Figure 2).

\section{Bringing patients and families together}

A patient camp taking place the next day was the forum for these patients and their families to come together, learn more about haemophilia, and start establishing a network of support. Information sessions were delivered in English and Ugandan, with Dr Khair and Professor Grace Ndezi delivering a haemophilia basics session, and physiotherapist Annette Nakalyango demonstrating simple exercises that could be done safely at home (Figure 3). Around 120 patients and family members attended the camp - including 46-year old Richard, the oldest known patient in Uganda. For many, the camp was the first time patients and families had been able to share their own experiences - some emotional, many inspiring - giving a sense of community, and often, a sense of hope. 


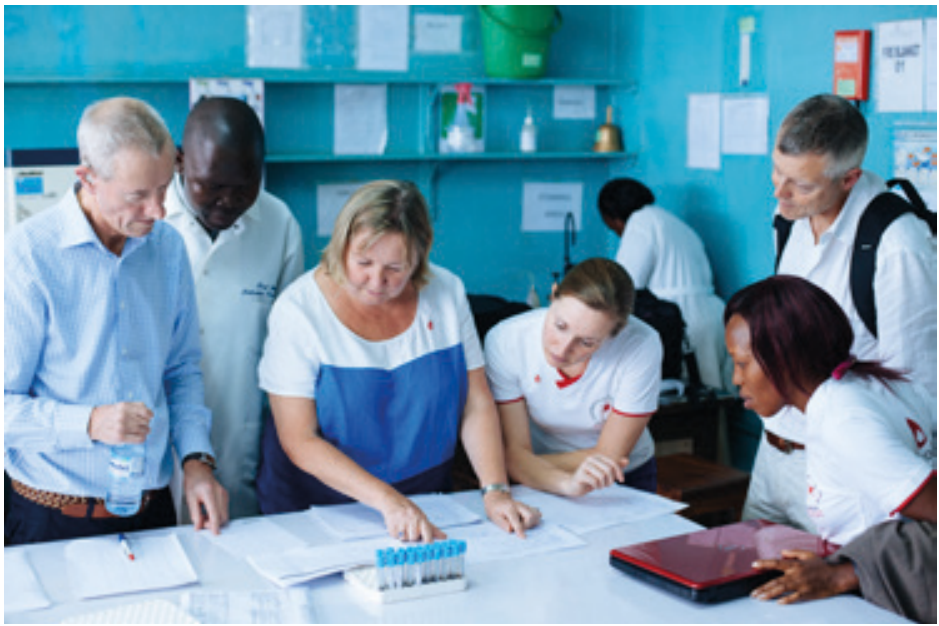

Figure 2: In arriving at a diagnosis, it was important to consider each lab result in the light of each individual clinical history

Using media to identify patients and engage authorities Alongside these activities to establish diagnosis and inform patients and families about haemophilia, activities were taking place to engage the national media in raising awareness of the condition.

An interactive two-day strategic media engagement workshop was delivered in September 2015 by the NNHF in collaboration with renowned Ugandan health journalist Irene Namyalo. Participants included patients, parents and healthcare professionals. Throughout the two days, the group gained practical experience of media interviews, and developed a media plan aimed at achieving the project's goals. The plan comprised two streams: the first was to identify potential patients through a television, radio and Facebook campaign; the second was to organise a press briefing during the screening day to reach out to the authorities.

The first part of the plan was put into action immediately, so that those identified through the media campaign as showing symptoms of haemophilia could be invited to be

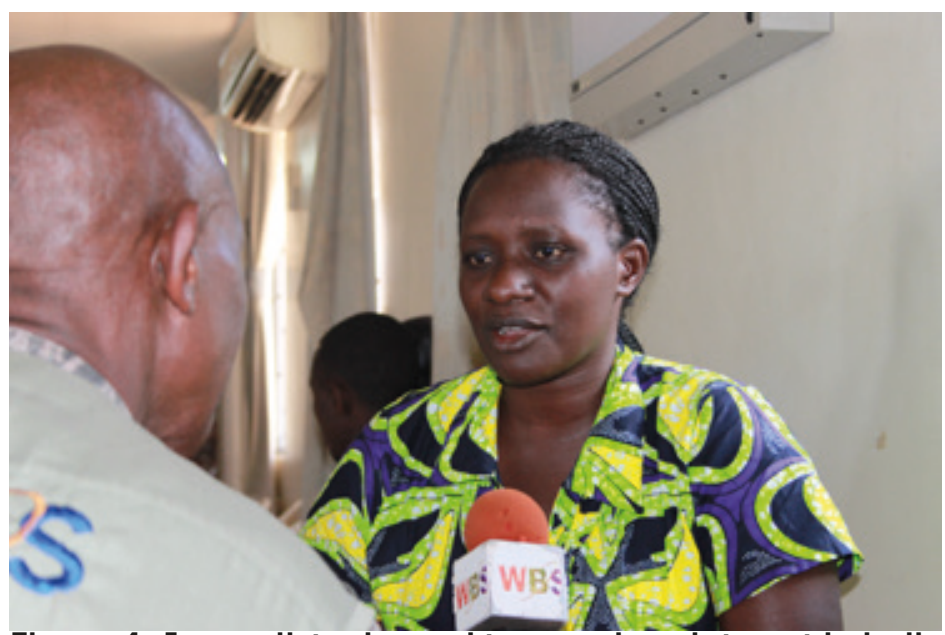

Figure 4: Journalists showed tremendous interest in individual patient stories

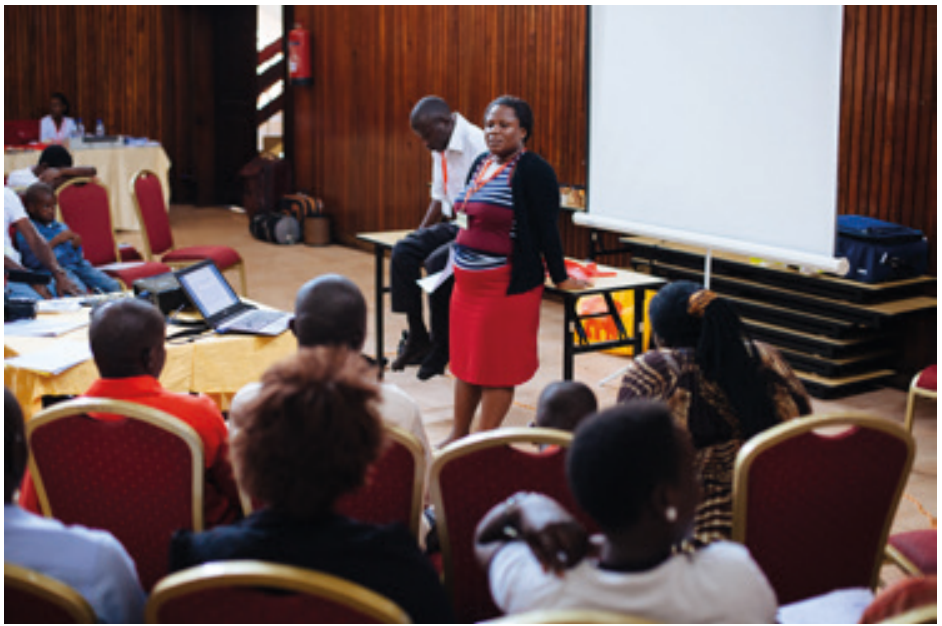

Figure 3: At the patient camp physiotherapist Annette Nakalyango demonstrating safe exercises that could be done at home

"I have received basic coagulation training before in South Africa, but the added benefit with the training delivered in Kampala by Dr Angus McCraw is that I am working alongside my fellow lab technicians and we can share experiences and questions - and we will continue to do so going forward"

Bwambale Joshua, a lab technician who also has haemophilia A

tested at December's screening day. Under the headline "Save lives by knowing", the team described the symptoms of haemophilia through a series of Facebook posts and radio and television interviews. They encouraged people showing these symptoms to contact the patient association. Those cases where haemophilia did seem the likely cause were invited to the screening day.

The second part of the media action plan culminated in a press briefing during the screening day itself. This time, the message to media was a call to authorities to fund reagents, so that diagnosis of haemophilia could continue as part of a national programme. The majority of those screened came from the area around Kampala, for many from further afield, the travelling alone was too much to come to screening.

In all, 27 journalists covered the event, with coverage appearing on two of Uganda's main news channels. Press interest was very high, with many journalists requesting an informational session about haemophilia, so they could create accurate stories targeted to the right audience (Figure 4).

"This is an excellent outcome for us and for all those affected by haemophilia in Uganda. We will continue to build on our partnership with authorities, and our next steps will be to expand care to the regions, establish comprehensive care at Mulago Hospital and identify more patients through a targeted media and education campaign"

Kisakye Agnes, executive secretary of the patient association and NNHF project partner 


\section{Bringing hope to Justine's family}

Nayiga Justine and her family (pictured right) offer an example of why this project is needed. A mother of eight, only one of her five sons had been diagnosed with haemophilia: two more boys showed symptoms, but had not been diagnosed as the family could not afford the fee for the test, which is currently only available in private labs and costs more than half the average Ugandan's monthly salary. Without a formal diagnosis no child is able to receive proper care.

With her husband working in Northern Uganda for weeks at a time, Justine was also unable to take her sons to the hospital when they had a bleed, as her only transport option was a motorcycle taxi. Due to long periods spent at home caused by constant joint bleeds, all three of her sons with haemophilia are behind with their education.

Through the NNHF project, Justine and her sons were able to travel to the screening day, where her two undiagnosed sons were tested and received their own diagnosis. At the patient camp that followed, Justine found out more about her sons' condition and how to cope with it better in daily life.

"Now that I have attended the patient camp and met other people with haemophilia, I have hope that my children will reach adulthood, and survive to be able to see their own grandchildren," said Justine.

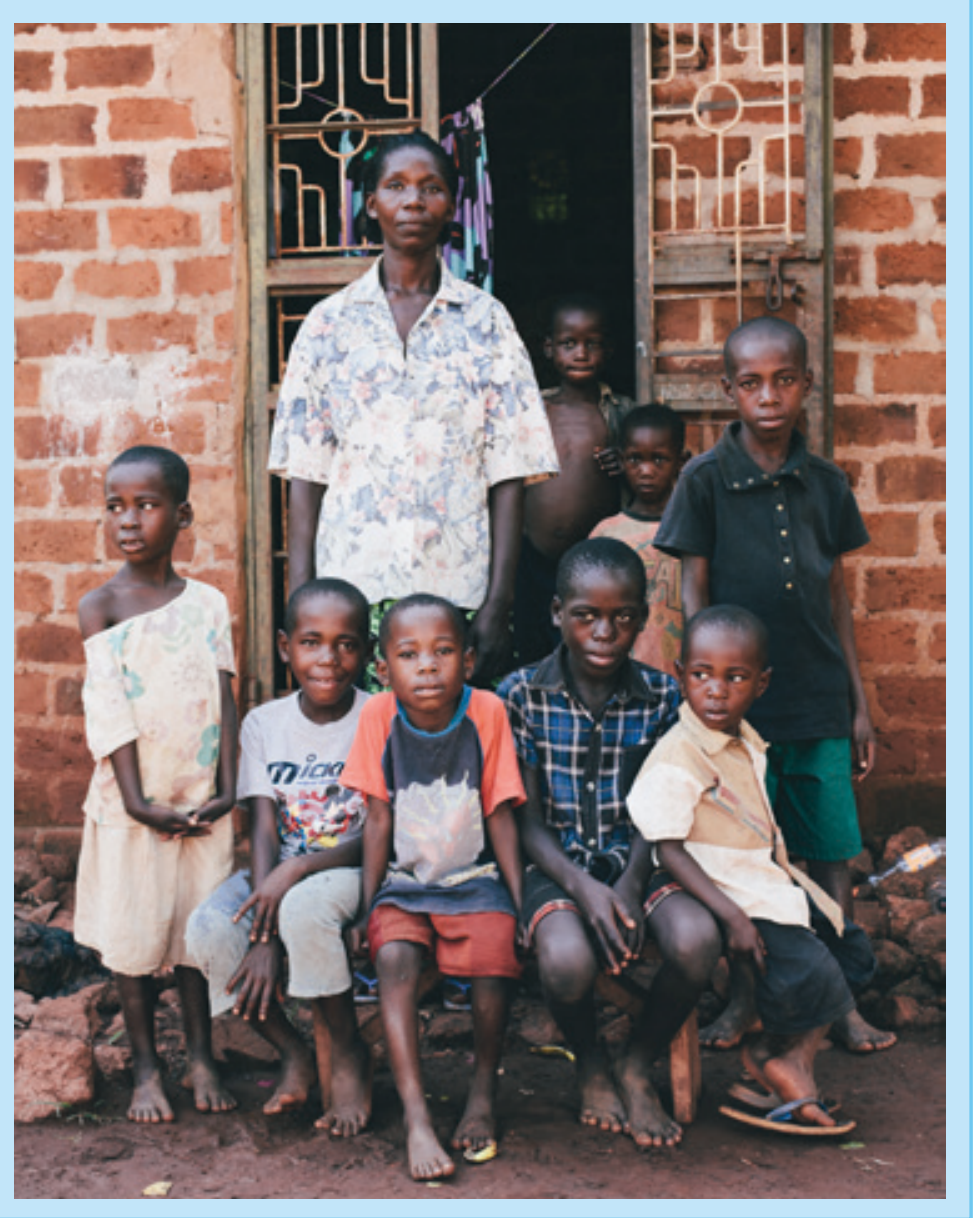

will apply the knowledge gained through the fellowship to support the objectives of the project.

\section{Disclosures}

The author has advised no interests that might be perceived as posing a conflict or bias.

This is an Open Access article distributed under the terms of the Creative Commons Attribution License (http:// creativecommons.org/licenses/by/2.0), which permits unrestricted use, distribution, and reproduction in any medium, provided the original work is properly cited.

\section{Acknowledgements}

Photographs are courtesy of William Leach Photography and the Novo Nordisk Haemophilia Foundation

Khair's team at Great Ormond Street Hospital in London. Dr Hilda, a member of the Uganda patient association,

Watch a short film about the NNHF Uganda project at http://novo.23video.com/secret/13390128/ f12e2fc8107a3a4881dfebf98bd21e0a

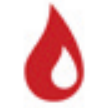

novo nordisk haemophilia foundation 